Peer-Reviewed Original Research

\section{Hemodynamic, Therapeutic, and Clinical Outcomes of Ambulatory Pressure-Guided Heart Failure Management}

\author{
Jacob Abraham*, Lian Wang, Rebecca Lewis, Katherine Callis, Joshua \\ Remick, and Kateri J. Spinelli
}

*Corresponding author: jacob.abraham@providence.org

Center for Cardiovascular Analytics, Research and Data Science (CARDS), Providence Heart Institute, Providence St. Joseph Health, Portland, OR

\begin{abstract}
Background

Heart failure (HF) management guided by CardioMEMS ${ }^{\mathrm{TM}}$ pulmonary artery pressure (PAP) monitoring reduces PAP and HF hospitalizations. The objective of this project was to characterize the relationship between medication adjustments, PAP change, and outcomes for all patients at an advanced HF center.
\end{abstract}

\section{Methods}

We retrospectively analyzed medication changes and hospitalizations for 32 consecutive patients implanted with the CardioMEMS ${ }^{\mathrm{TM}}$ sensor at a single HF center and related these outcomes to PAP data from the Merlin.net (Abbott) database. Absolute change in PAP from baseline was estimated using area under the curve normalized to days monitored. 


\section{Results}

Patients had an average change in mean PAP of $-3.9 \pm 1.3 \mathrm{mmHg}$ at 6 months and $-5.7 \pm 2.1 \mathrm{mmHg}$ at 12 months. Compared to the pre-implant time period, hospital days for HF-related hospitalizations decreased by $6.9(95 \% \mathrm{Cl}=3.3-10.5)$ days per patient at 6 months $(\mathrm{p}<0.001)$ and $7.9(95 \% \mathrm{Cl}=4.3-11.5)$ days per patient at 12 months $(p<0.001)$. Over 12 months, patients with baseline PAP 25-35mmHg and $>35 \mathrm{mmHg}$ experienced a drop in PAP while their number of medication adjustments decreased, and patients with baseline PAP $<25 \mathrm{mmHg}$ experienced a rise in PAP and an increase in medication adjustments.

\section{Conclusion}

PAP-guided HF management involved time-varying intensity of medication changes, which correlated with sustained reductions in hospital stay.

Keywords: heart failure, hemodynamics, pulmonary artery, CardioMEMS, outcomes

\section{Introduction}

Despite advances in pharmacologic and device therapies, heart failure (HF) remains a progressive syndrome associated with high rates of hospitalization and mortality, poor quality of life, and staggering health care costs. ${ }^{1}$ An important hemodynamic feature of HF is elevation of the left ventricular end-diastolic pressure that can lead to secondary elevation of pulmonary artery pressure. ${ }^{2}$ Regardless of the left ventricular ejection fraction, pressure elevation is closely associated with symptom burden, hospitalizations, and mortality. ${ }^{3-5}$ Strategies to manage HF guided by non-invasive surrogates of hemodynamics have been unsuccessful. $6,7,8$

In the CHAMPION (CardioMEMS ${ }^{\text {TM }}$ Heart Sensor Allows Monitoring of Pressure to Improve Outcomes in NYHA Class III Heart Failure Patients) trial, HF management guided by ambulatory pulmonary artery pressure (PAP) monitoring using a wireless implantable sensor (CardioMEMS ${ }^{\text {TM }}$ Heart Failure System, Abbott) decreased HF-related hospitalizations six months after implant, compared to control patients receiving standard care. ${ }^{9}$ Following commercial approval of the sensor, real world studies showed that CardioMEMS ${ }^{\mathrm{TM}}$ implantation is associated with meaningful reductions in PAP and, separately, HF hospitalizations. ${ }^{10,11}$

Although ambulatory hemodynamic monitoring has shown promise, the optimal use and implementation in routine clinical practice remains to be defined. Significant uncertainty remains over patient selection, the frequency and intensity of medication adjustment required to affect PAP, the relationship at a patient level between PAP change and clinical outcomes, and the magnitude and durability of clinical benefit. These uncertainties have lead to calls for comprehensive, real world studies to fully assess the impact of this device. ${ }^{12}$ 
In the present study, we report our experience with the CardioMEMS ${ }^{\mathrm{TM}}$ system at a non-transplant advanced HF program operating within a large health care system. We examine changes in PAP for up to 12 months following CardioMEMS ${ }^{\mathrm{TM}}$ implant and relate longitudinal PAP changes to baseline hemodynamics, medication adjustment, and clinical outcomes.

\section{Methods}

\section{Patients and data source}

Patients selected for implant with the CardioMEMS ${ }^{\mathrm{TM}}$ sensor met the following criteria: New York Heart Association (NYHA) class III chronic heart failure; at least one HF-related hospitalization in the year prior to implant; life expectancy $>1$ year; GFR $>25 \mathrm{ml} / \mathrm{min} / 1.73 \mathrm{~m}^{2}$; responsive to diuretics; expected to be adherent to daily pressure monitoring and staff instructions; and could be reliably contacted by telephone. According to institutional practice, patients were eligible only after management by the institution's board-certified HF cardiologists to achieve maximally tolerated medical and device therapy per professional guidelines. The CardioMEMS ${ }^{\mathrm{TM}}$ Heart Failure System (Abbott, Sylmar, California) was then implanted by one of two HF specialists and managed by the same HF team, comprised of 2 HF physicians and 2 clinic nurses, according to institution-specific general management guidelines. The study was approved by the Providence Health \& Services Oregon institutional review board, with waiver of informed consent.

Between January 2015 and December 2016, 32 patients received a CardoMEMS ${ }^{\mathrm{TM}}$ sensor at our facility. We performed chart reviews of these consecutive patients to collect demographic and baseline hemodynamic data, as well as hospitalization history one year prior to the implant and medication adjustments post implant. The study cutoff date for retrospective chart review was $2 / 20 / 2017$. A request to retrieve the PA pressure data for these patients from the Abbott Merlin.net database was submitted. A copy of the PA pressure data was harvested in May 2017 when the request was approved; thus the pressure dataset includes an additional 2-3 months of data beyond the hospitalizations and medication adjustments dataset. Pressure data contains the date and time of each pressure reading transmission, along with derived pressure measures (mean, systolic, and diastolic PAP). No patients were lost to follow-up.

\section{Pressure management}

Following sensor implant, an initial mean PAP goal of $<25 \mathrm{mmHg}$ was targeted. Patients' pressures were then monitored every 2 to 3 days on Merlin.net by a registered nurse. In general, adjustments in medications were made with approval from a supervising HF cardiologist based on a center-specific treatment protocol (Appendix), in conjunction with lab testing, patient symptoms, and clinical judgment. Adjustments in medication were communicated to patients by phone or in person, if during a clinic visit. 


\section{Statistical Analysis}

The effectiveness of the pressure monitoring in managing the PAP was assessed using the area under the curve (AUC) methodology described by Heywood et al. ${ }^{10}$ The pressure data from the first week after implant was used as the baseline. A time series of PAP was generated for each patient, with linear interpolation between data points. The AUC was computed for the area between the PAP timeline and baseline PAP for each patient, starting either from the time of implant to the desired time point or within the time interval of a selected time window (e.g. 3-6 months). Because AUC is a measure of cumulative change ( $\mathrm{mmHg}$-day) and is time-interval duration dependent, we further calculated the pressure change from baseline for each patient by dividing AUC by the number of days in the time interval (normalized change in PAP, $\mathrm{mmHg}$ ). For subgroup analyses, patients were categorized by baseline mean PAP $(<25 \mathrm{mmHg}, 25-35 \mathrm{mmHg}$, and $>35$ $\mathrm{mmHg}$ ), gender, or left ventricle ejection fraction (LVEF) status, defined as HF with preserved ejection fraction (HFpEF) $=\mathrm{EF} \geq 40 \%$ and HF with reduced ejection fraction $(\mathrm{HFrEF})=\mathrm{EF}<40 \%$. Both AUC and change in PAP were reported as mean \pm SE.

The clinical outcomes of all-cause and HF-related hospitalizations (planned and unplanned) were compared pre- and post-implant. For a selected time window (e.g., 6-12 months), only patients with post-implant follow-up time long enough for this window were included in the pre- vs. post-implant comparison. The average number of days for all-cause and HF-related hospitalizations within a certain time window pre- and post-implant was compared using paired t-tests. Rates of hospitalization within 12 months pre- and post-implant were compared using the Anderson-Gill regression modeling for recurrent events, taking into account withinsubject correlations with robust variance estimate. ${ }^{13}$ One patient who died at 2.4 months after the implant was excluded in the hospitalization rate analysis; LVAD patients were included in the analysis. Subgroup analyses for hospitalization rate were performed for patients grouped by baseline PAP, gender, LVEF status, and age $<75$ years old. Due to the small sample sizes in the subgroups, the time window for subgroup analyses was limited to 6 months.

Total medication adjustments within drug classes during the study period were reported; types of adjustments involving loop diuretics were further characterized. To explore the relationship between medication adjustments and PAP changes over time, the average change in PAP for each of the three baseline mean PAP groups in the time windows of 0-3, 3-6, 6-9, and 9-12 months post implant, were calculated and graphed as a function of time. The average number of medication adjustments for each baseline PAP group in the corresponding time interval was calculated and the PAP markers were sized relative to this number. Medication adjustments over time and change in PAP over time was also graphed for individual patients, grouped by baseline PAP. In these graphs, closed markers indicate that the patient had an HF-related hospitalization in the time window and open markers indicate an absence of HF-related hospitalization. To avoid bias in these calculation due to incomplete follow-up data, only patients with long enough follow-up time for both medication adjustments and pressure data to cover the selected time window were included in that time window. The R statistical program 
(www.r-project.org, R Foundation for Statistical Computing, Vienna, Austria) was used for all analyses. Figures were created in Adobe Illustrator CC 2014.

\section{Results}

\section{Patient population}

From January 2015 through December 2016, 32 patients were implanted with CardioMEMS $^{\mathrm{TM}}$ at our center (Table 1). The average age was 66 years old, $50 \%$ were female, and the majority of patients (62.5\%) had HFrEF. The majority of patients had comorbidities and laboratory data that indicated mild to moderate renal insufficiency. Patients in our clinic were actively managed for HF prior to CardioMEMS implant, as indicated by the high number of HFrEF patients prescribed evidence-based therapies at baseline.

\section{Table 1: Patient demographics}

\begin{tabular}{|c|c|}
\hline Variable & Values \\
\hline \# of patients implanted & 32 \\
\hline Age, years, mean $\pm S D$, median (range) & $66.6 \pm 10.2,66(40-88)$ \\
\hline Gender, female, n (\%) & $16(50 \%)$ \\
\hline Race, white, n (\%) & $30(93.8 \%)$ \\
\hline $\mathrm{BMI}, \mathrm{kg} / \mathrm{m}^{2}$, mean $\pm \mathrm{SD}$ & $33.8 \pm 9.5$ \\
\hline Preserved EF, n (\%) & $13(40.6 \%)$ \\
\hline \multicolumn{2}{|l|}{ Comorbidities, n (\%) } \\
\hline Coronary artery disease & $18(56.3 \%)$ \\
\hline ICD or pacemaker & $16(50 \%)$ \\
\hline Diabetes mellitus & $17(53.1 \%)$ \\
\hline Lung disease & $21(65.6 \%)$ \\
\hline \multicolumn{2}{|l|}{ Laboratory data, mean \pm SD } \\
\hline Sodium & $137.9 \pm 3.2$ \\
\hline BUN & $36.9 \pm 22.0$ \\
\hline Creatinine & $1.47 \pm 0.47$ \\
\hline \multicolumn{2}{|l|}{ Baseline hemodynamics, mean \pm SD } \\
\hline Right atrial pressure, $\mathrm{mmHg}$ & $12.6 \pm 7.1$ \\
\hline Cardiac index, L/min $/ \mathrm{m}^{2}$ & $2.63 \pm 0.81$ \\
\hline Cardiac output, L/min & $5.54 \pm 2.10$ \\
\hline
\end{tabular}


Baseline PAP, n (\%)

$$
<25 \mathrm{mmHg}
$$

$25-35 \mathrm{mmHg}$

$\geq 35 \mathrm{mmHg}$

Medications, for HFrEF patients*, n (\%)

Beta blocker

ACEI or ARB or ARNI

$10(91 \%)$

Spironolactone

$6(55 \%)$

Implant approach, $\mathrm{n}(\%)$

Femoral vein

Internal jugular vein

Follow-up time, months, median (range)

$12.6(2.3-25.0)$

* Excluding 8 patients with stage $4-5$ chronic kidney disease $(n=5)$ and VAD patients $(n=3)$

$\mathrm{ACEI}=$ angiotensin-converting enzyme inhibitor, $\mathrm{ARB}=$ angiotensin receptor blockers , $\mathrm{ARNI}=$ angiotensin receptor-neprilysin inhibitor, $\mathrm{BMI}=$ body mass index, $\mathrm{BUN}=$ blood urea nitrogen, $\mathrm{EF}=$ ejection fraction, $\mathrm{HFrEF}=$ heart failure reduced ejection fraction, $\mathrm{ICD}=$ implantable cardioverter defibrillator, $\mathrm{PAP}=$ pulmonary artery pressure, $\mathrm{SD}=$ standard deviation

Eighty-one percent of patients had baseline PAP above normal (>25 $\mathrm{mmHg}$ ) and $41 \%$ had baseline PAP >35 mmHg. The majority of patients $(60 \%)$ were implanted via the internal jugular vein. The median follow-up time was 12.6 months. Three patients treated with durable left ventricular assist devices (LVAD) at least 12 months prior to sensor implant were included; exclusion of these patients did not impact the major results from any analyses, thus these patients were included to increase sample size. There were no procedure-related, device-related, or systemrelated complications.

One patient deteriorated and underwent LVAD implantation two months after sensor implant. One patient died two and a half months after implant from combined septic/cardiogenic shock. An additional five deaths occurred after one year post-implant, with causes of death including renal failure, progressive heart failure, out-of-hospital cardiac arrest, bowel perforation, and cause unknown.

\section{Pulmonary artery pressure changes}

Cumulative changes in PAP over time were measured using the area under the curve (AUC) method previously described (Supplemental Figure 1). ${ }^{10}$ We further calculated normalized AUC, which represents the absolute change in mean PAP 
from baseline, which can easily be compared across different time-interval lengths. For the overall population, average change in PAP was $-3.9 \pm 1.3 \mathrm{mmHg}$ at 6 months and $-5.7 \pm 2.1 \mathrm{mmHg}$ at 12 months (Figure $1 \mathrm{~A}$ ). Patients with the highest baseline PAP (>35 $\mathrm{mmHg}$ ) had the greatest drop in pressure at 12 months $(-10.4 \pm$ $3.5 \mathrm{mmHg}$, compared to baseline), while patients with the lowest baseline PAP $(<25 \mathrm{mmHg})$ had a slight increase in pressure at 12 months $(2.0 \pm 3.0 \mathrm{mmHg}$, compared to baseline; Figure 1B).
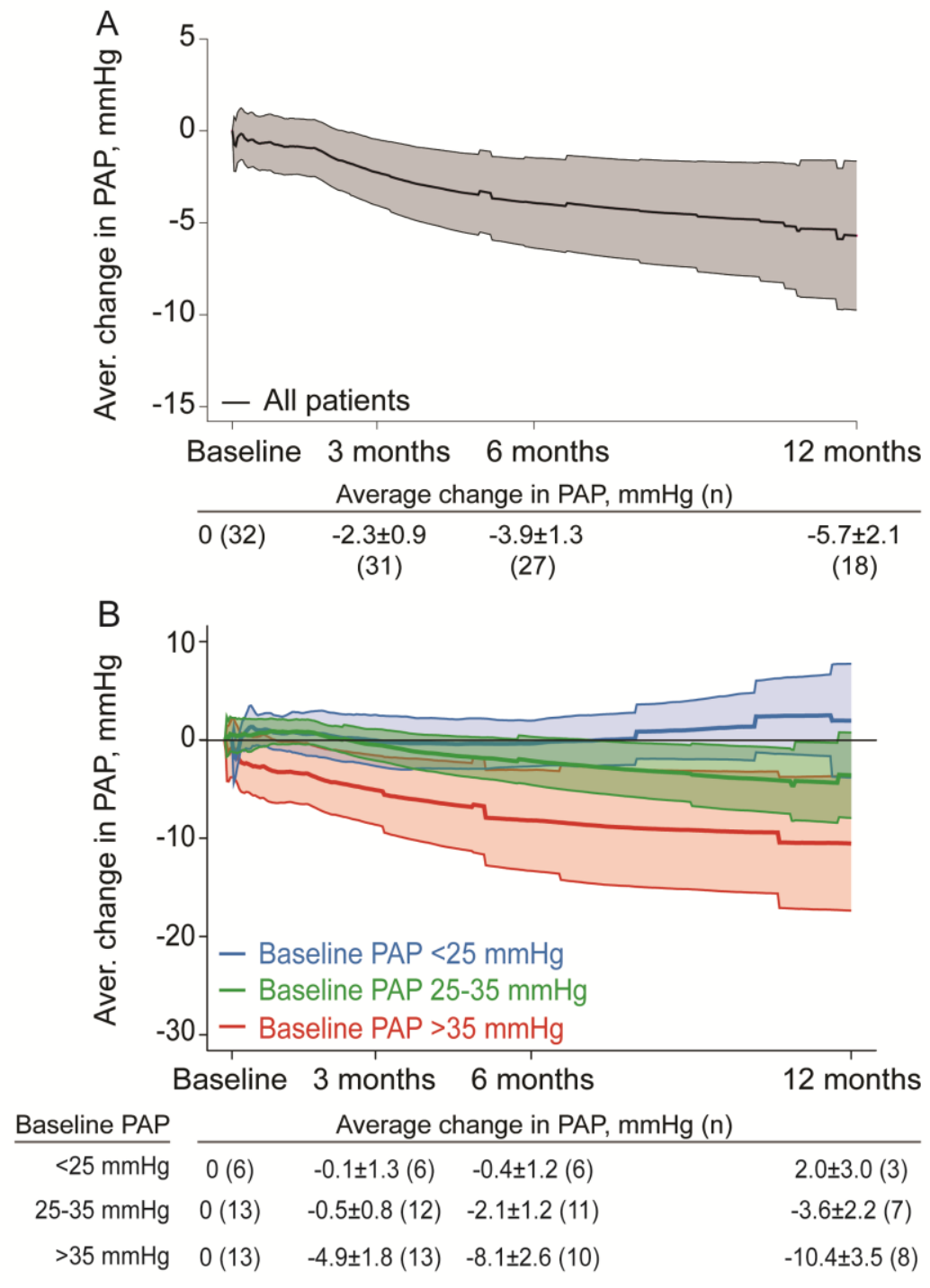

Figure 1: Changes in pulmonary artery pressures over time. Absolute pressure change over time, reported as mean PAP AUC normalized by days monitored per patient, averaged for the overall population $(A)$ and for each baseline pressure group (B). For both graphs, solid line indicates average pressure and shaded area indicates $95 \%$ confidence intervals. Data tables report mean \pm SE (number of patients). $A U C=$ area under the curve, PAP $=$ pulmonary artery pressure. 
Sustained PAP reductions over time were observed in both male and female patients and in patients with reduced and preserved EF (Supplemental Figure 2). There was no difference in baseline PAP between HFrEF and HFpEF patients $(\mathrm{HFpEF}$ mean $\pm \mathrm{SD}=32.5 \pm 13.7$, HFrEF mean $\pm \mathrm{SD}=35.2 \pm 9.0, \mathrm{p}=0.56)$.

\section{Hospitalizations}

Cumulative incidence of HF-related hospitalization pre- and post-implant is presented in Figure 2. For the overall patient population, sensor implant was associated with a $72 \%$ reduction in the risk of HF-related hospitalizations at 12 months (hazard ratio (HR) [95\% confidence interval] $=0.28$ [0.14-0.55]; $p=0.002$ ). The reduction in HF-related hospitalization at 6 months post-implant, compared to the pre-implant time period, was significant when patients were stratified by baseline PAP, gender, EF status, and age.
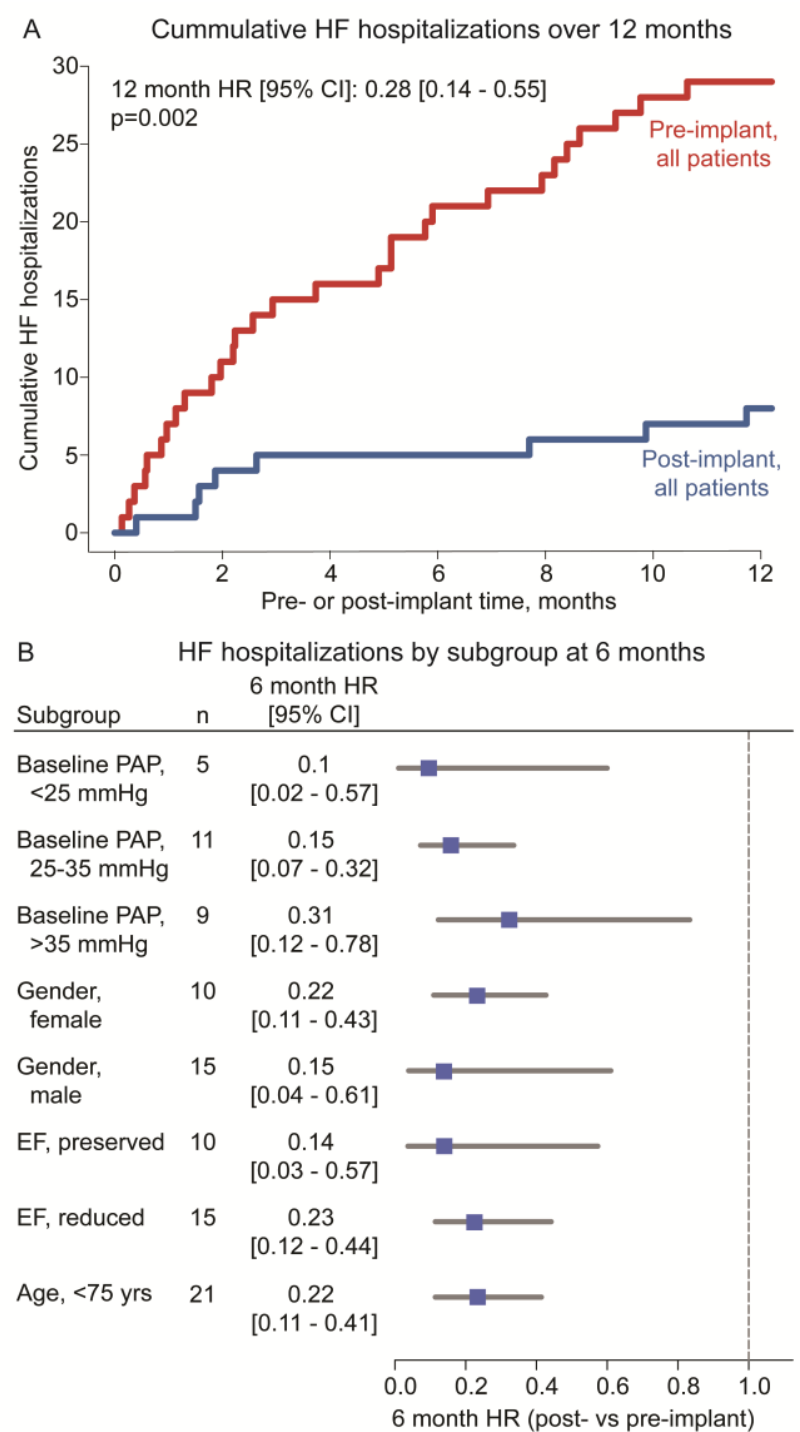

Figure 2 - Reduction in cumulative HF-related hospitalizations. (A) Sustained decrease in cumulative HF-related hospitalizations up to 12 months post-implant for all patients $(n=17)$. (B) 6-month hazard ratios for postvs pre-implant HF-related hospitalizations by subgroup $(n=25) . \mathrm{Cl}=$ confidence interval, $\mathrm{EF}=$ ejection fraction, $\mathrm{HF}=$ heart failure, $\mathrm{HR}$ $=$ hazard ratio, $\mathrm{PAP}=$ pulmonary artery pressure. 
Figure 3 shows the pre- and post-implant timeline of hospitalizations, grouped into 3-month intervals. The average number of hospital days for all-cause and HFrelated hospitalizations increased during the pre-implant time period, peaking in the 3 months prior to implantation. Following sensor implantation, there was an immediate decrease in the number of hospital days for all-cause and HF-related hospitalizations, which was sustained over one year. Compared to the pre-implant time period, hospital days for all-cause hospitalizations decreased by 7.4 days per patient $(95 \% \mathrm{Cl}=2.3-12.6 ; \mathrm{p}=0.007)$ and hospital days for HF-related

hospitalizations decreased by 6.9 days per patient $(95 \% \mathrm{Cl}=3.3-10.5 ; \mathrm{p}<0.001)$ at 6 months post-implant (Table 2). At 12 months post-implant, hospital days for allcause hospitalizations decreased by 7.2 days per patient $(95 \% \mathrm{Cl}=2.2-12.2$; $\mathrm{p}=0.008$ ) and hospital days for HF-related hospitalizations decreased by 7.9 days per patient $(95 \% \mathrm{Cl}=4.3-11.5 ; \mathrm{p}<0.001)$, compared to the pre-implant time period (Table 2).
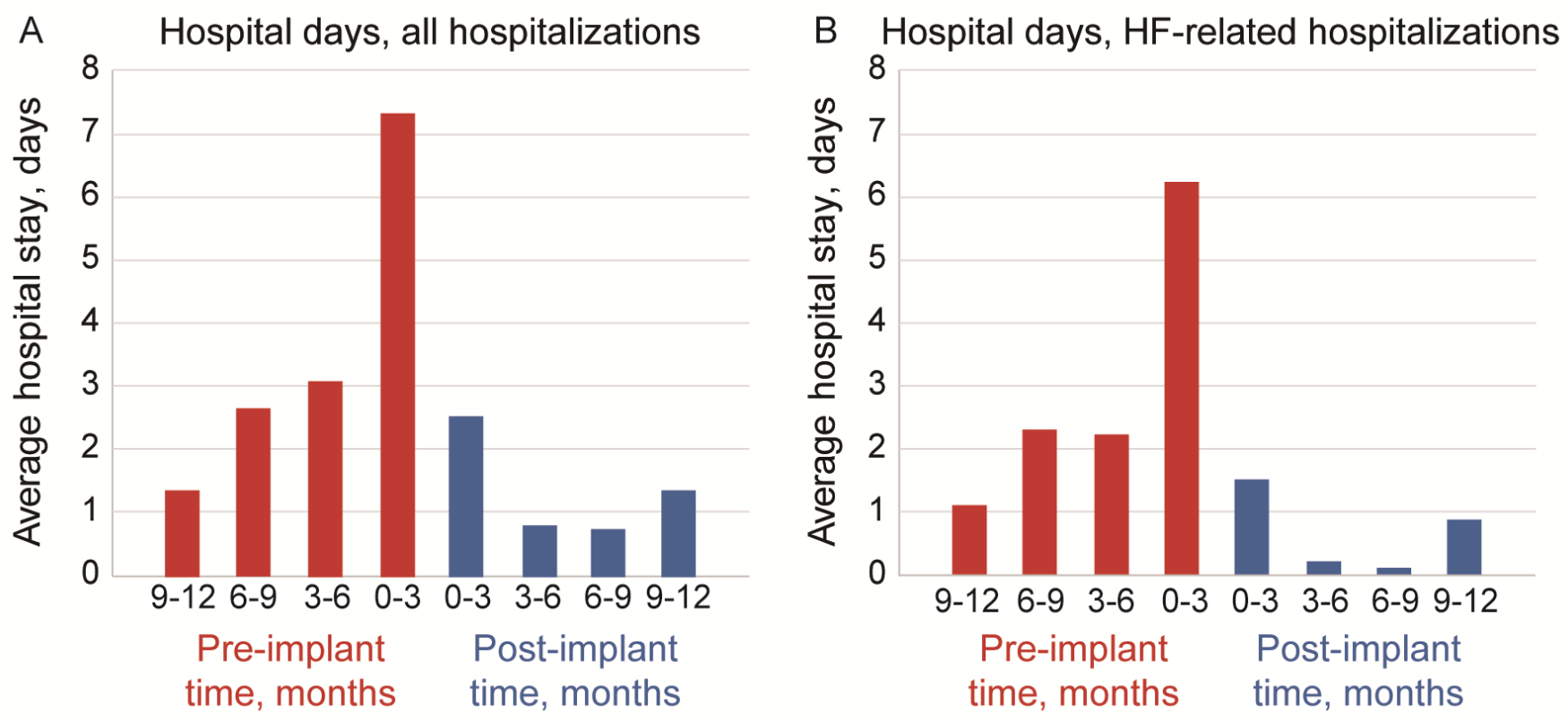

Figure 3: Significant decrease in hospital days post-implant. Time line of the average number of hospital days for all-cause $(A)$ and HF-related (B) hospitalizations pre- (red) and post-implant (blue).

\section{Adherence and medication adjustments}

Over the study period, patients transmitted data 9,959 times over 12,370 patientdays, for an average of one transmission every 1.24 days. Time between transmissions was 0.96 days in the first 30 days post-implant, 1.12 days in 6 months post-implant, and 1.15 days in one year post-implant. Median utilization, defined as the percentage of weeks with at least one transmission, ${ }^{9}$ was $100 \%$ $(\mathrm{IQR}=96.1-100 \%)$ at 6 months follow up and $97.7 \%(\mathrm{IQR}=94.6-100 \%)$ at one year follow up. 
Table 2: Hospital days for all-cause and HF-related hospitalizations, pre- and post-implant

\begin{tabular}{|c|c|c|c|c|c|c|}
\hline \multirow{2}{*}{$\begin{array}{l}\text { Pre/post } \\
\text { implant time } \\
\text { frame }\end{array}$} & \multicolumn{4}{|c|}{ All-cause hospitalizations } & \multirow{2}{*}{$\begin{array}{l}\text { Reduction } \\
\text { (95\% Cl) }\end{array}$} & \multirow[b]{2}{*}{ p-value } \\
\hline & $\begin{array}{l}\text { Total days, } \\
\text { pre-implant }\end{array}$ & $\begin{array}{l}\text { Total days, } \\
\text { post-implant }\end{array}$ & $\begin{array}{c}\text { Days per } \\
\text { patient, } \\
\text { pre-implant }\end{array}$ & $\begin{array}{c}\text { Days per } \\
\text { patient, } \\
\text { post-implant }\end{array}$ & & \\
\hline $\begin{array}{l}6 \text { months } \\
(n=25)\end{array}$ & 270 & 84 & 10.8 & 3.4 & $\begin{array}{c}7.4 \\
(2.3-12.6)\end{array}$ & 0.007 \\
\hline $\begin{array}{l}12 \text { months } \\
(n=17)\end{array}$ & 211 & 89 & 12.4 & 5.2 & $\begin{array}{c}7.2 \\
(2.2-12.2)\end{array}$ & 0.008 \\
\hline \multirow{2}{*}{$\begin{array}{l}\text { Pre/post } \\
\text { implant time } \\
\text { frame }\end{array}$} & \multicolumn{4}{|c|}{ HF-related hospitalizations } & \multirow[b]{2}{*}{$\begin{array}{c}\text { Reduction } \\
\text { (95\% Cl) }\end{array}$} & \multirow[b]{2}{*}{ p-value } \\
\hline & $\begin{array}{l}\text { Total days, } \\
\text { pre-implant }\end{array}$ & $\begin{array}{l}\text { Total days, } \\
\text { post-implant }\end{array}$ & $\begin{array}{c}\text { Days per } \\
\text { patient, } \\
\text { pre-implant }\end{array}$ & $\begin{array}{c}\text { Days per } \\
\text { patient, } \\
\text { post-implant }\end{array}$ & & \\
\hline $\begin{array}{l}6 \text { months } \\
(n=25)\end{array}$ & 222 & 49 & 8.9 & 2.0 & $\begin{array}{c}6.9 \\
(3.3-10.5)\end{array}$ & $<0.001$ \\
\hline $\begin{array}{l}12 \text { months } \\
(\mathrm{n}=17)\end{array}$ & 189 & 55 & 11.1 & 3.2 & $\begin{array}{c}7.9 \\
(4.3-11.5)\end{array}$ & $<0.001$ \\
\hline
\end{tabular}

$\mathrm{Cl}=$ confidence interval

There were 282 medication adjustments over the study period, with an average of 1.5 patient-months between adjustments. The average number of medication adjustments per patient per month was $0.82 \pm 0.65$ for the overall patient population and less than one for each of the baseline PAP subgroups. The majority of medication adjustments were in loop diuretics $(53.9 \%, 152$ adjustments) and other diuretics (16.3\%, 46 adjustments) (Supplemental Figure 3). For loop diuretics, 48.3\% (75 adjustments) were increases in dose and 30.3\% (46 adjustments) were decreases.

\section{Relationship between pressure changes, medication adjustments, and hospitalizations}

We stratified patients according to baseline PAP ( $<25 \mathrm{~mm} \mathrm{Hg}, 25-35 \mathrm{~mm} \mathrm{Hg}$, and $>35 \mathrm{~mm} \mathrm{Hg}$ ) and analyzed the relationship between pressure changes over time and medication adjustments (Figure 4). Patients with low baseline PAP $(<25$ $\mathrm{mmHg}$ ) had an average of 1.8 and 2.0 medication adjustments for the first $0-3$ and 3-6 months post-implant, respectively, which rose to 5.0 medication adjustments at 9-12 months post-implant. This group experienced an average increase in PAP of $4.8 \mathrm{mmHg}$ at 9-12 months post-implant. Conversely, patients with the highest 
pressures at baseline ( $>35 \mathrm{mmHg}$ ) had an average of 3.5 medication adjustments at 0-3 months, but only required 1.3 medication adjustments at 9-12 months. These patients experienced an average drop in PAP of $5.1 \mathrm{mmHg}$ in the first $0-3$ months and $11.5 \mathrm{mmHg}$ at months 9-12. Patients with intermediate baseline PAP $(25-35 \mathrm{mmHg})$ started out with the greatest average number of medications adjustments (4.3) in the first 0-3 months but required the smallest average number of adjustments (1.1) at 9-12 months. These patients experienced an average drop in PAP of $6.4 \mathrm{mmHg}$ at 9-12 months post-implant.

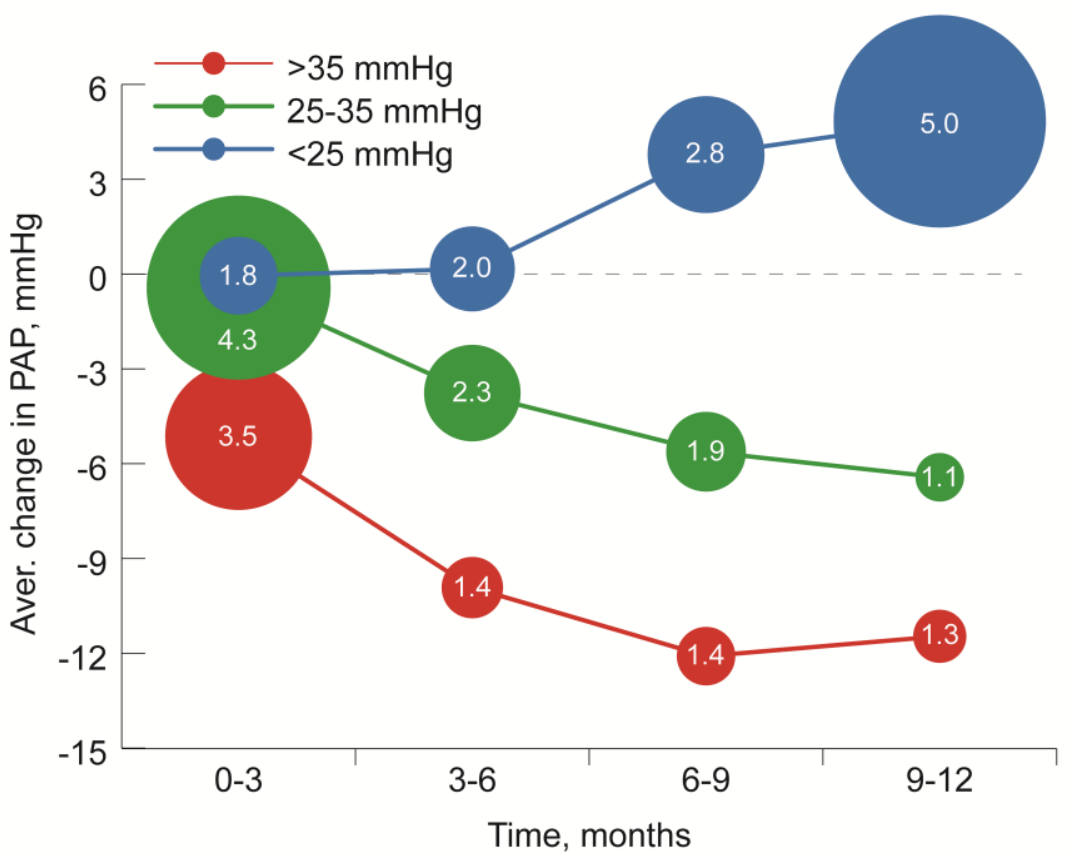

Figure 4 - Average pressure change and medication adjustments over time for each baseline pressure group. The average number of medication adjustments for each pressure group at each time point is included within the marker, and the marker size is scaled accordingly. AUC = area under the curve, $\mathrm{PAP}=$ pulmonary artery pressure.

Supplemental Figure 4 provides patient-level data on medication adjustments, pressure changes, and HF-related hospitalizations for patients grouped by baseline pressure. Patients with $>35 \mathrm{mmHg}$ baseline PAP experienced more HFrelated hospitalizations at multiple time points post-implant. Conversely, patients with baseline normal PAP experienced an increase in PAP yet did not require hospitalization. Notably, several patients in all three pressure groups experienced no change in PAP over time, despite increased medication adjustments.

\section{Discussion}

In this cohort of consecutive patients implanted with a CardioMEMS ${ }^{\mathrm{TM}}$ sensor at a single advanced HF center, we found that device implant was associated with 
significant overall reductions in PA pressure. Although variability was observed in individual pressure changes, patients with abnormal baseline PAP $(>25 \mathrm{mmHg})$ experienced reductions in PAP while the number of medication adjustments decreased over time, whereas patients with normal baseline PAP experienced a rise in PAP and required more medication adjustments over time. Consistent with randomized and observational studies, we found a substantial reduction in cumulative HF-related hospitalizations at 6 and 12 months post-implant compared to the pre-implant period. Adding to these previous studies, we report a reduction of 7.2 days per patient for all-cause and 7.9 days per patient for HF hospitalizations at 12 months after implant.

In this cohort, the majority of all-cause hospitalizations in the pre-implant time frame were HF-related, which is reflected in the reduction in the number and days of all-cause hospitalizations post-implant. The decrease in HF-related hospitalizations did not result in an increase in non-HF-related hospitalizations. The reduction in all-cause hospitalization is in agreement with previous analyses. ${ }^{11}$ Of considerable interest, number of hospital days for all-cause and HF-related hospitalization was dramatically reduced after sensor implant. There are several potential explanations for these findings. First, patients readmitted with HF were likely to be hospitalized in an earlier stage of decompensation given longitudinal knowledge of PA pressure. Secondly, CardioMEMS patients can have their sensor interrogated during hospitalization at the discretion of the managing physician. It is therefore possible that knowledge of changes in PAP during hospitalization impacted the decision of when to discharge. Finally, ambulatory hemodynamic management of HF can improve common co-morbidities in HF patients. ${ }^{14}$ Non-HF admissions for common comorbidities could conceivably be shorter as a result of improvement in HF and ability to interrogate PA pressure.

This is the first study to report patient-level interactions between hemodynamics, medication adjustments, and clinical outcomes. Although the absolute reductions in PA pressure observed in the studied population are numerically modest, the association with substantial clinical benefit (e.g. fewer hospitalizations) is consistent with an analysis by Zile et al which showed that even a $5 \mathrm{mmHg}$ reduction in PA diastolic pressure is associated with a $30 \%$ mortality reduction. ${ }^{15}$ Costanzo et al reported that patients with higher baseline PA diastolic pressures had more medication adjustments than those with lower baseline pressures. ${ }^{16}$ In contrast, our patients in the highest baseline pressure stratum had fewer medication adjustments over time, particularly at 6-12 months post-implant as pressures plateaued. Conversely, in the small cohort of patients with normal baseline pressures, more medication changes were required over time. These findings likely reflect the approach at our center to attempt aggressive lowering of PAP in the first few months after implant.

We found that despite multiple medication adjustments, some patients in each pressure stratum had no change in PAP, and for those in the highest pressure stratum, inability to reduce pressure correlated with an increased risk of hospitalization. Some of these patients had cardiorenal limitations to PAPlowering, and we speculate that others likely had irreversible pulmonary hypertension indicative of more advanced HF. The role of acute vasodilator 
studies in predicting hemodynamic and clinical responsiveness to ambulatory, pressure guided HF management is not known.

A strength of our analysis compared to previous real world publications is that patients were selected for sensor implant only after optimization of standard medical therapy by HF specialists. Continuity of care through the same HF specialty clinic pre- and post-implant reduces the likelihood that under treatment of HF prior to implant, rather than PAP-guidance, was the source of benefit. Patients with HFrEF were treated with maximal medical therapy and device therapy, as tolerated. Additionally, following implant, patients in this study were managed according to a nurse-implemented management algorithm with HF physician oversight. Management by a limited team of providers using an empiric algorithm (Appendix) reduces variability in treatment approach and strengthens the generalizability of our findings.

Included in this analysis are 4 patients with continuous flow left ventricular assist devices (LVAD) implanted as destination therapy. One patient experienced progressive deterioration and required durable LVAD implantation 2 months after sensor implant; due to concern about the patient's candidacy for an LVAD, the sensor was implanted with the intention of preventing or delaying the need for surgery. We successfully managed three other LVAD patients who had persistent NYHA class III symptoms following LVAD implant. In total, all four LVAD patients had improved hemodynamics and reduced hospitalizations following CardioMEMS ${ }^{\mathrm{TM}}$ implantation, consistent with another cases series. ${ }^{17}$ Further research is needed to optimize selection and management of patients with advanced co-morbid conditions and prior to and after LVAD implantation.

An analysis of medical claims data from 1114 patients implanted with CardioMEMS $^{\mathrm{TM}}$ in a real-world setting found a $34 \%$ reduction in $\mathrm{HF}$ hospitalizations at one year, which correlated to cost reductions of $\$ 11,260$ per patient. ${ }^{11}$ We could therefore reasonably expect significant cost-savings associated with our results of a $72 \%$ reduction in HF hospitalizations at one year post-implant. Cost-effectiveness analysis was outside of the scope of this study; however, our workload analysis suggests that with less than one medication adjustment per patient per month, CardioMEMS ${ }^{\mathrm{TM}}$ monitoring is feasible and scalable as part of a population health strategy for HF.

There were no complications associated with device implantation. Of interest, 34\% of patients were implanted as outpatients via the right internal jugular vein. Although this access route is not mentioned in the CardioMEMS ${ }^{\mathrm{TM}}$ sensor FDA approval, it has become the preferred approach at our center and allows for continuation of anticoagulation, earlier patient mobilization post-procedure, and faster time to discharge. ${ }^{18}$

This study has a number of limitations. First, the overall sample size is small and drawn from a single-center. Sample size notwithstanding, our findings are statistically and clinically significant, concordant with the results of larger studies, and reflect treatment of a real-world, representative HF patient population. Second, as a pre-/post-implant design is employed, we cannot exclude the potential for selection bias or confounding by other HF interventions that were 
applied in the post-implant interval. However, because all patients were managed by the same experienced HF team before and after implant, it is less likely there were significant differences in treatment before and after sensor implant. Third, the physicians implanting and managing patients were HF specialists experienced with pressure-guided HF management, potentially limiting generalizability. This limitation is minimized by the use of a treatment algorithm that was implemented by clinic nursing. Finally, while we report the number of medication adjustments as a reflection of clinical workload, we are not able to measure the additional time and effort needed for reviewing pressure data, contacting patients, troubleshooting the device, and educating patients.

\section{Conclusions}

This analysis of a single center experience with hemodynamic-guided HF management using an implantable sensor shows that improvements in hemodynamics and clinical outcomes are achievable with an acceptable degree of workload for clinical staff. Further studies are needed to optimize patient selection and to assess cost-effectiveness.

\section{Acknowledgments}

We thank Rupinder Bharmi, MS, for feedback on data analysis.

\section{References}

1. Benjamin EJ, Blaha MJ, Chiuve SE, et al. Heart Disease and Stroke Statistics-2017 Update: A Report From the American Heart Association. Circulation. 2017;135:e146-e603.

2. Braunwald E. Heart failure: pathophysiology and treatment. American Heart Journal. 1981;102:486-90.

3. Drazner MH, Rame JE, Stevenson LW and Dries DL. Prognostic importance of elevated jugular venous pressure and a third heart sound in patients with heart failure. The New England Journal of Medicine. 2001;345:574-81.

4. Lucas C, Johnson W, Hamilton MA, et al. Freedom from congestion predicts good survival despite previous class IV symptoms of heart failure. American Heart Journal. 2000;140:840-7.

5. Stevenson LW, Zile M, Bennett TD, et al. Chronic ambulatory intracardiac pressures and future heart failure events. Circulation: Heart Failure. 2010;3:580-7.

6. Lewin J, Ledwidge M, O'Loughlin C, McNally C and McDonald K. Clinical deterioration in established heart failure: what is the value of BNP and weight gain in aiding diagnosis? European Journal of Heart Failure. 2005;7:953-7.

7. Felker GM, Anstrom KJ, Adams KF, et al. Effect of Natriuretic PeptideGuided Therapy on Hospitalization or Cardiovascular Mortality in High-Risk Patients With Heart Failure and Reduced Ejection Fraction: A Randomized Clinical Trial. JAMA. 2017;318:713-720. 
8. Chaudhry SI, Mattera JA, Curtis JP et al. Telemonitoring in patients with heart failure. NEJM 2010;363:2301-9.

9. Abraham WT, Adamson PB, Bourge RC, et al. Wireless pulmonary artery haemodynamic monitoring in chronic heart failure: a randomised controlled trial. Lancet. 2011;377:658-66.

10. Heywood JT, Jermyn R, Shavelle D, et al. Impact of Practice-Based Management of Pulmonary Artery Pressures in 2000 Patients Implanted With the CardioMEMS Sensor. Circulation. 2017;135:1509-1517.

11. Desai AS, Bhimaraj A, Bharmi R, et al. Ambulatory Hemodynamic Monitoring Reduces Heart Failure Hospitalizations in "Real-World" Clinical Practice. Journal of the American College of Cardiology. 2017;69:2357-2365.

12. Krumholz HM and Dhruva SS. Championing Effectiveness Before CostEffectiveness. JACC: Heart Failure. 2016;4:376-9.

13. Andersen PK and Gill RD. Cox's Regression Model for Counting Processes: A Large Sample Study. The Annals of Statistics. 1982;10:1100-1120.

14. Krahnke JS, Abraham WT, Adamson PB, et al. Heart failure and respiratory hospitalizations are reduced in patients with heart failure and chronic obstructive pulmonary disease with the use of an implantable pulmonary artery pressure monitoring device. Journal of Cardiac Failure. 2015;3:240-9.

15. Zile MR, Bennett TD, El Hajj S, et al. Intracardiac Pressures Measured Using an Implantable Hemodynamic Monitor: Relationship to Mortality in Patients With Chronic Heart Failure. Circulation: Heart Failure. 2017;10.

16. Costanzo MR, Stevenson LW, Adamson PB, et al. Interventions Linked to Decreased Heart Failure Hospitalizations During Ambulatory Pulmonary Artery Pressure Monitoring. JACC: Heart Failure. 2016;4:333-44.

17. Guglin M, George B, Branam S and Hart A. CardioMEMS ${ }^{\mathrm{TM}}$ in LVAD Patients: A Case Series. The VAD Journal. 2016;2.

18. Abraham J, Lewis RS, Wang L, et al. Under Pressure: When to Stick Your Neck Out? JACC: Heart Failure. 2017;5:156. 